\title{
Correction: A blood-based prognostic biomarker in IBD
}

Biasci D, Lee JC, Noor NM, et al. A blood-based prognostic biomarker in IBD. Gut $2019 ; 68: 1386-95$.

Figures 1-4 of this article were not printed in colour in the August issue of Gut in error.

A

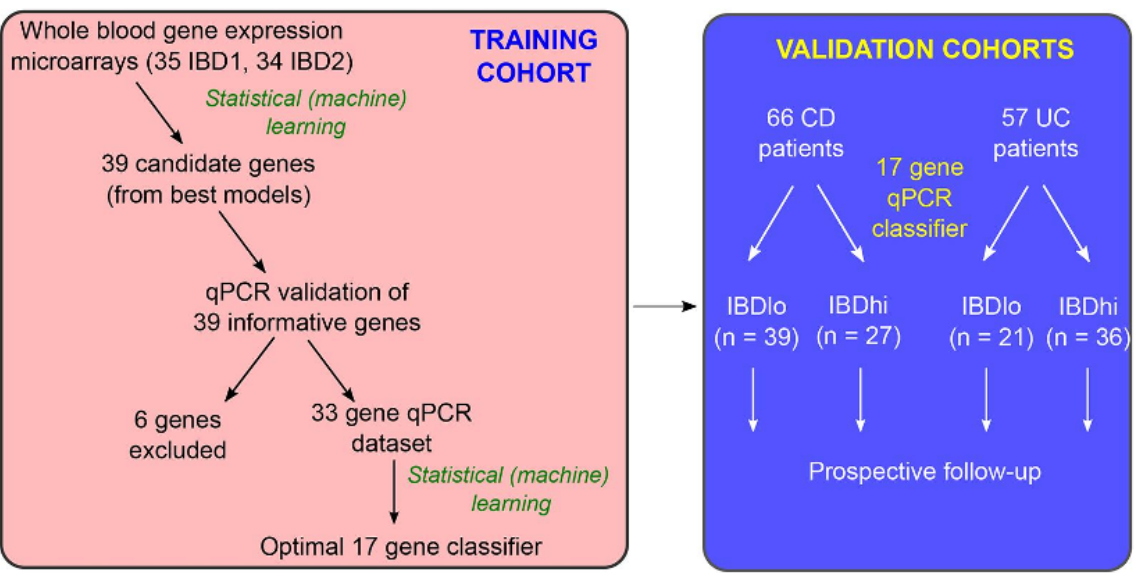

B

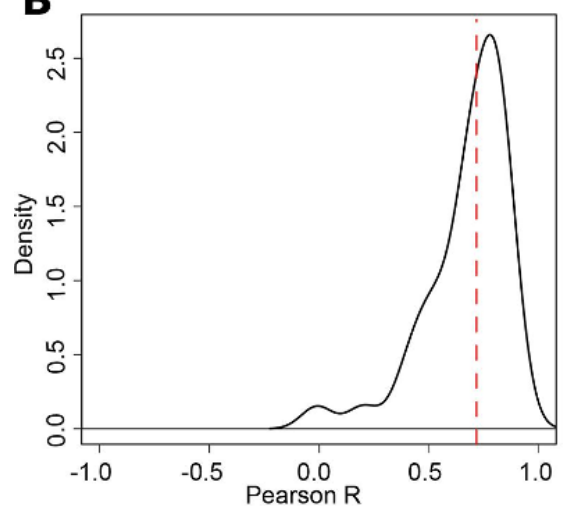

C

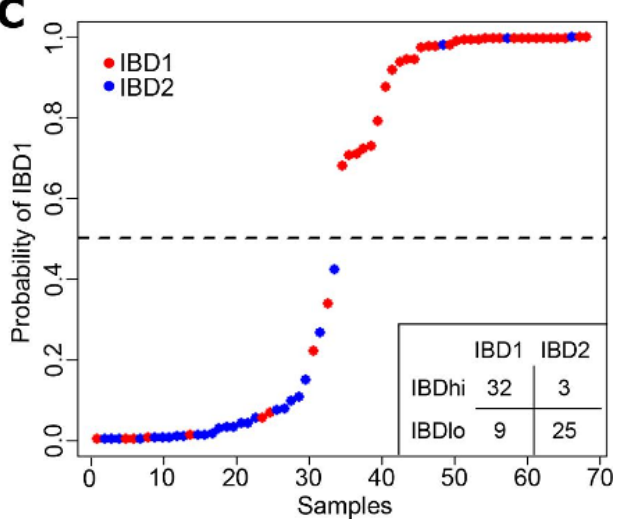

Figure 1 Development of a qPCR-based whole blood prognostic biomarker. (A) Schematic depicting the workflow for the development, optimisation and validation of the whole blood qPCR-based classifier with separate training and validation cohorts. (B) Distribution of correlation coefficients between microarray and qPCR-based measurements of gene expression for 39 genes. (C) Confidence of assignments to IBD1 and IBD2 subgroups in the training cohort using the $\mathrm{qPCR}$ classifier (15 informative and 2 reference genes). Colours indicate actual IBD1/IBD2 assignments based on CD8 T cell transcriptomic analysis (red=IBD1, blue=IBD2). Inset summary table depicts results using 0.5 cut-off for group assignment. $C D$, Crohn's disease. 
A

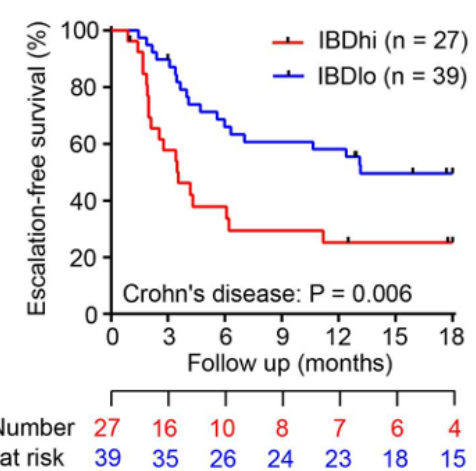

C
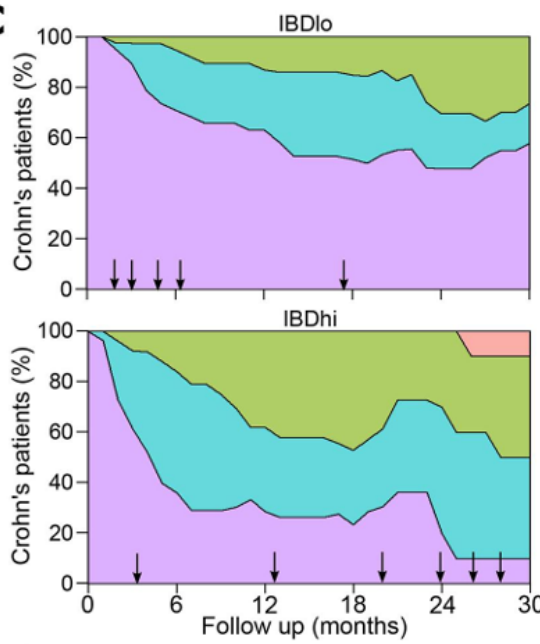

No treatment $\square$ Anti-TNFa

$\square$ Immunomodulator $\square$ 2nd line biologic

$\mathbf{E}$

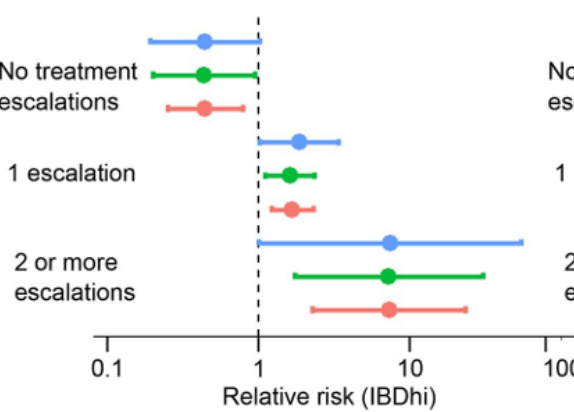

B

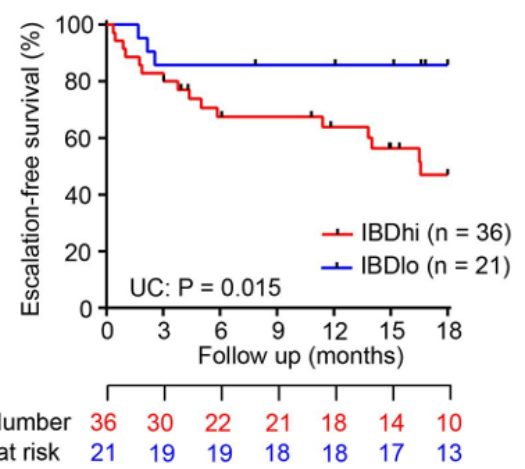

D
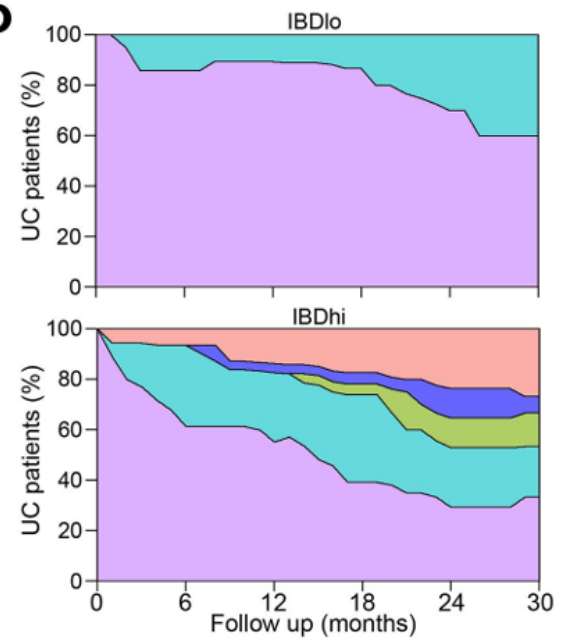

5-ASA only $\square$ Anti-TNFa $\square$ Colectomy Immunomodulator $\square$ Vedolizumab

$\mathbf{F}$

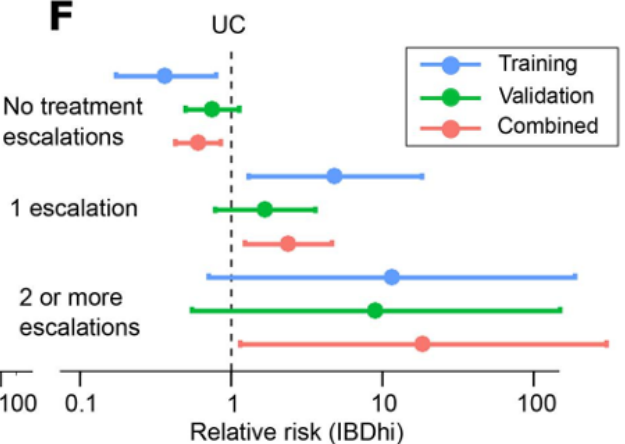

Figure 2 Validation of qPCR-based classifier in independent cohorts. (A and B) Kaplan-Meier plots of escalation-free survival for the $C D$ validation cohort $(A ; n=66)$ and the $U C$ validation cohort $(B ; n=57)$ as stratified by the IBDhi (IBD1 equivalent) and IBDlo (IBD2 equivalent) patient subgroups. Data are censored at 18 months. Statistical significance assessed by log-rank test. (C and D) Stacked density plots demonstrating the maximum medical therapy that was required during the first 2.5 years' prospective follow-up of the IBDhi and IBDlo subgroups in CD (C) and UC (D). Treatments were plotted hierarchically (no treatment<immunomodulator $<$ anti-TNF $\alpha<$ second-line biologicals (vedolizumab or ustekinumab) in CD and 5-ASA only<immunomodulator $<$ anti-TNF $\alpha<$ vedolizumab $<$ colectomy in UC). Arrows represent episodes of surgery that were required for CD patients at the indicated timepoints. Data are censored accordingly to length of follow-up so that the denominator is the total available cohort at each timepoint. ( $E$ and F) Forest plots of the relative risk (IBDhi vs IBDlo) of requiring no treatment escalations, one treatment escalation or two or more treatment escalations within the first 18 months after enrolment for patients with $C D(E)$ and patients with UC (F). Relative risk is with respect to the IBDhi subgroup in each disease and is presented separately for the training cohort, validation cohort and combined cohorts. Error bars indicate $95 \%$ Cls. CD, Crohn's disease. 
A

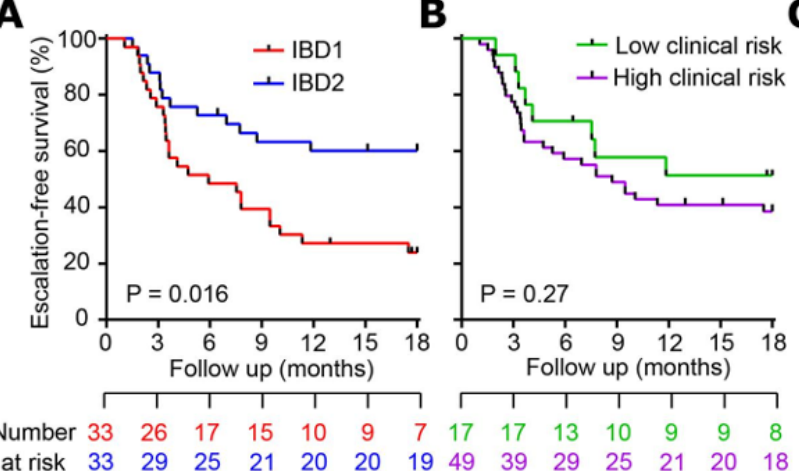

C_ No severe

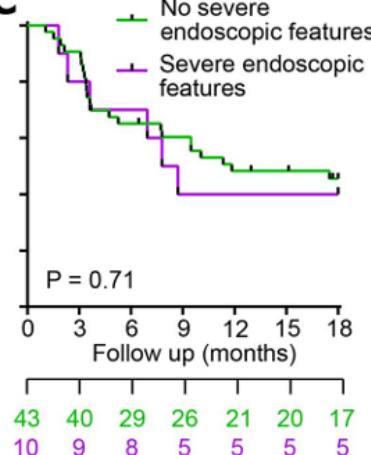

D
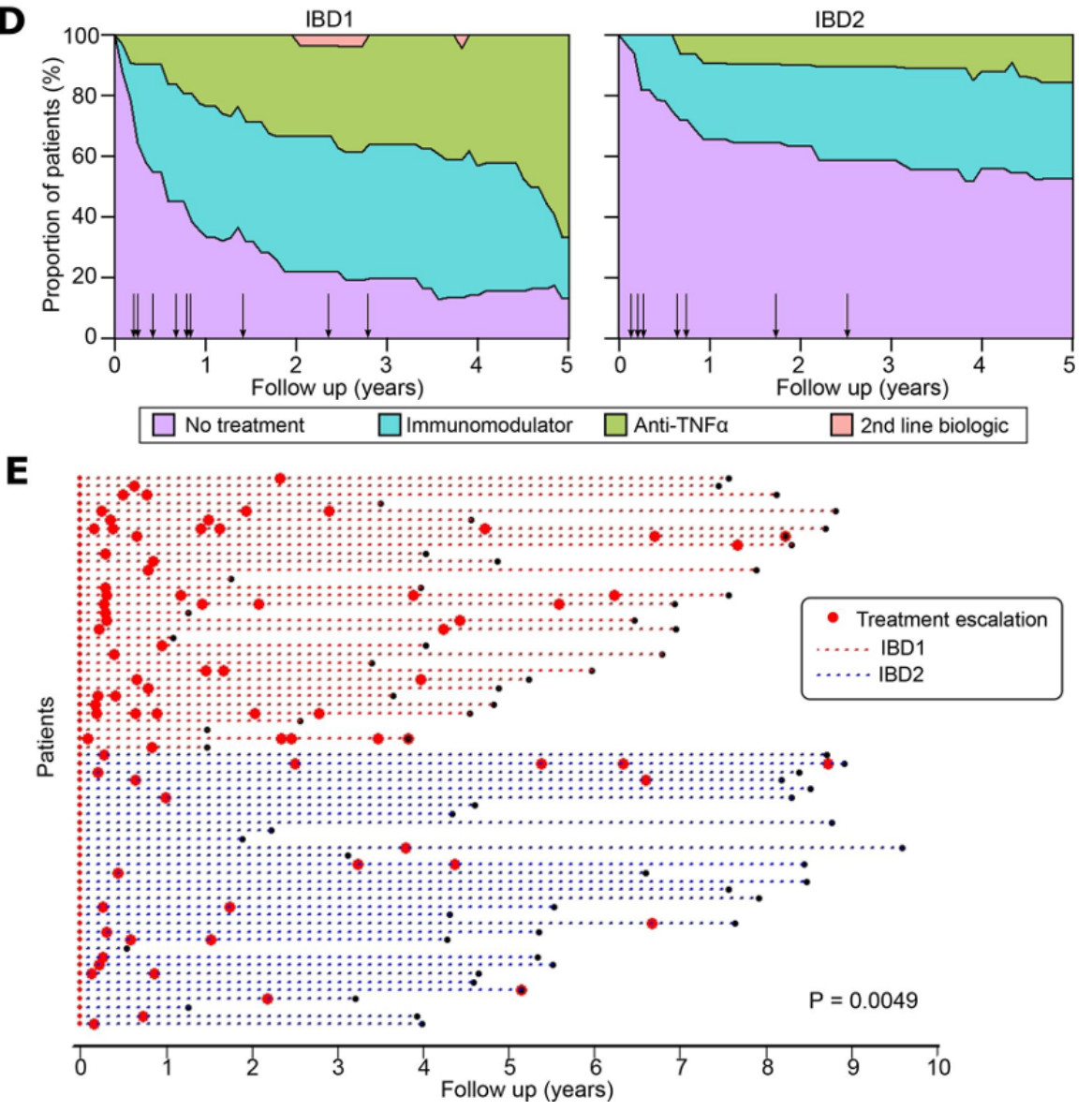

Figure 3 The clinical course of Crohn's disease (CD) is different in IBD1 and IBD2 patients. (A) KaplanMeier plot of escalation-free survival for CD patients in the IBD1 and IBD2 subgroups. Data are censored at 18 months. Statistical significance assessed by log-rank test. (B and C) Kaplan-Meier plots in the same format as figure part A with patients subdivided according to clinical risk (high risk=2 or more of: age $<40$ years at diagnosis, early need for steroids and perianal disease; B) and presence of severe features at index endoscopy (deep and extensive ulceration in at least one colonic segment or endoscopist's global assessment; C). (D) Stacked density plots demonstrating the maximum medical therapy that was required during 5 years' prospective follow-up in the IBD1 and IBD2 subgroups. Treatments were plotted hierarchically (no treatment<immunomodulator<antiTNF $\alpha<$ second-line biologicals (vedolizumab or ustekinumab)). Arrows represent episodes of surgery that were required at the indicated timepoints (of note one operation that is indicated in online supplementary table 4-in an IBD1 patient-occurred after 5 years and is not shown). Data are censored accordingly to length of follow-up so that the denominator is the total available cohort at each timepoint. (E) Disease course of individual CD patients (dotted lines). The colour of dotted lines reflects subgroup designation. Statistical significance was determined using a Mann-Whitney test. 
A

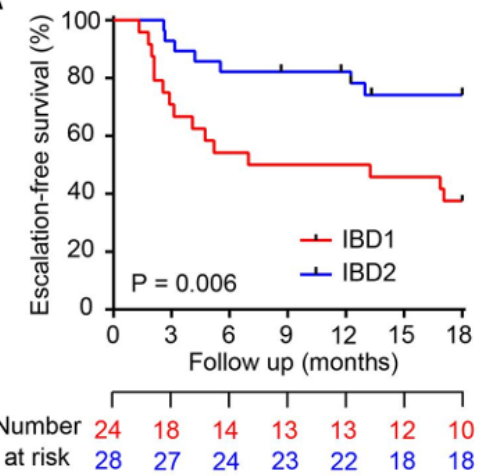

B

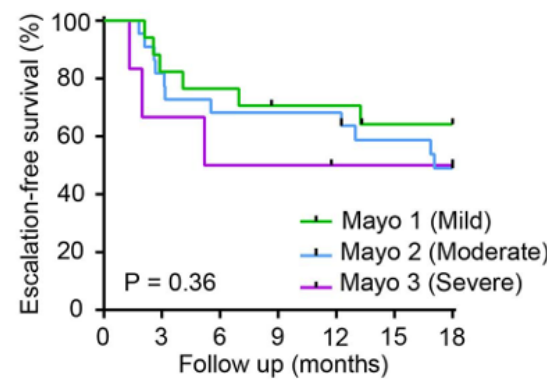

C

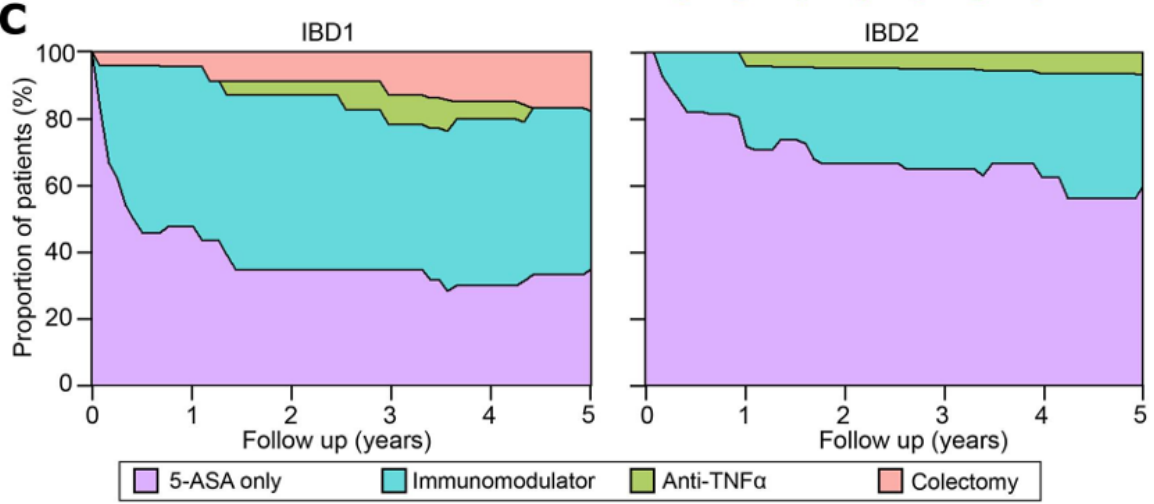

D

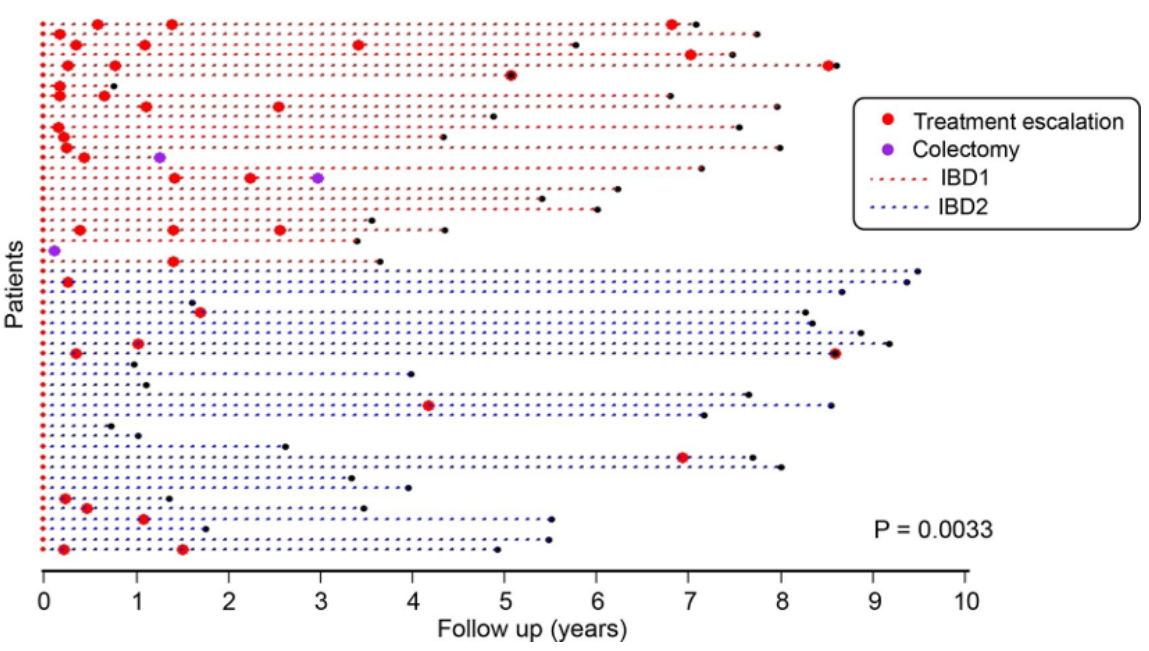

Figure 4 The clinical course of UC is different in IBD1 and IBD2 patients. (A) Kaplan-Meier plot of escalation-free survival for UC patients in the IBD1 and IBD2 subgroups. Data are censored at 18 months. Statistical significance assessed by log-rank test. (B) Kaplan-Meier plot in the same format as figure part A with patients subdivided according to endoscopic disease severity at index colonoscopy. $P$ value calculated by comparing mild and severe cases. (C) Stacked density plots demonstrating the maximum medical therapy that was required during the first 5 years' prospective followup in the IBD1 and IBD2 subgroups. Treatments were plotted hierarchically (5-ASA only<immunomodulator<antiTNF $\alpha<$ vedolizumab<colectomy). Data are censored accordingly to length of follow-up so that the denominator is the total available cohort at each timepoint. (D) Disease course of individual UC patients (dotted lines). The colour of dotted lines reflects subgroup designation. Statistical significance was determined using a MannWhitney test.

(C) Author(s) (or their employer(s)) 2019. No commercial re-use. See rights and permissions. Published by BMJ.

Gut 2019;68:1909-1912. doi:10.1136/gutjnl-2019-318343corr1

D) Check for updates 